(2) Open Access Full Text Article

\title{
Interactive navigation-guided ophthalmic plastic surgery: the utility of 3D CT-DCG-guided dacryolocalization in secondary acquired lacrimal duct obstructions
}

\author{
This article was published in the following Dove Press journal: \\ Clinical Ophthalmology \\ 30 December 2016 \\ Number of times this article has been viewed
}

\section{Mohammad Javed Ali \\ Swati Singh' \\ Milind N Naik' \\ Swathi Kaliki² \\ Tarjani Vivek Dave'}

'Govindram Seksaria Institute of Dacryology, ${ }^{2}$ The Operation Eyesight Universal Institute for Eye Cancer, LV Prasad Eye Institute, Hyderabad, Telangana, India
Correspondence: Mohammad Javed Ali Govindram Seksaria Institute of Dacryology, L V Prasad Eye Institute, Road No 2, Banjara Hills, Hyderabad 500034, Telangana, India Email drjaved007@gmail.com

\begin{abstract}
Aim: The aim of this study was to report the preliminary experience with the techniques and utility of navigation-guided, 3D, computed tomography-dacryocystography (CT-DCG) in the management of secondary acquired lacrimal drainage obstructions.

Methods: Stereotactic surgeries using CT-DCG as the intraoperative image-guiding tool were performed in 3 patients. One patient had nasolacrimal duct obstruction (NLDO) following a complete maxillectomy for a sinus malignancy, and the other 2 had NLDO following extensive maxillofacial trauma. All patients underwent a 3D CT-DCG. Image-guided dacryolocalization (IGDL) was performed using the intraoperative image-guided StealthStation ${ }^{\mathrm{TM}}$ system in the electromagnetic mode. All patients underwent navigation-guided powered endoscopic dacryocystorhinostomy (DCR). The utility of intraoperative dacryocystographic guidance and the ability to localize the lacrimal drainage system in the altered endoscopic anatomical milieu were noted.
\end{abstract}

Results: Intraoperative geometric localization of the lacrimal sac and the nasolacrimal duct could be easily achieved. Constant orientation of the lacrimal drainage system was possible while navigating in the vicinity of altered endoscopic perilacrimal anatomy. Useful clues with regard to modifications while performing a powered endoscopic DCR could be obtained. Surgeries could be performed with utmost safety and precision, thereby avoiding complications. Detailed preoperative 3D CT-DCG reconstructions with constant intraoperative dacryolocalization were found to be essential for successful outcomes.

Conclusion: The 3D CT-DCG-guided navigation procedure is very useful while performing endoscopic DCRs in cases of secondary acquired and complex NLDOs.

Keywords: CT-dacryocystography, image guidance, navigation, lacrimal drainage, nasolacrimal duct obstruction, powered endoscopic DCR

\section{Introduction}

In recent times, we are seeing an increased interest in the use of interactive imageguided surgeries for complex lacrimal drainage disorders. ${ }^{1-3}$ The advantages of stereotactic guidance, also referred to as image or navigation guidance, include increased anatomical orientation, accurate target localization and trajectory information, minimal collateral damage, enhanced safety and surgeon comfort during lacrimal procedures, specifically the endoscopic approaches. Computed tomography (CT)-dacryocystography (DCG) is a well-established modality of lacrimal imaging and is useful in delineating the lacrimal drainage system from the surrounding tissues 
and assessing the stenosis, dilatation or mass lesion within the lacrimal ducts. ${ }^{4-7}$ Although it is not routinely required in primary dacryocystorhinostomy (DCR) and routine secondary acquired nasolacrimal duct obstruction (SALDO) cases, it may be of value in cases of complex SALDO. The 3D reconstruction of the CT-DCG has been reported to allow the surgeon to view images in multiple projections and to enhance the accuracy of diagnosing pathologies. ${ }^{4}$ The authors of this study report their preliminary experiences with navigation guidance for lacrimal drainage surgeries using the $3 \mathrm{D}$ CT-DCG as the basic guiding tool.

\section{Methodology}

Approval from the institutional review board of L V Prasad Eye Institute was obtained. Patient consents for both the surgery and the publication of their reports and photos were obtained. Three consecutive patients with complex secondary acquired NLDO were included in this study. Complex SALDO was defined as a case of SALDO in which the lacrimal and the perilacrimal soft tissue or bony anatomy was grossly altered as per CT, or when routine bony landmarks were unavailable for intraoperative guidance for localization of the sac. Stereotactic surgeries were performed in these 3 patients using 3D CT-DCG, thereby enabling the highlighted lacrimal sac as the radiologic landmark and as the intraoperative image-guiding tool. All patients underwent a 3D CT-DCG. Image-guided dacryolocalization (IGDL) was performed using the intra-operative image-guided StealthStation ${ }^{\mathrm{TM}}$ system in the electromagnetic mode using the AxiEMTM technology. All patients underwent navigation-guided powered endoscopic DCR. The utility of intraoperative DCG guidance and the ability to localize the lacrimal drainage system in the altered endoscopic anatomical milieu were noted.

For intraoperative navigation, contiguous CT scans of $1 \mathrm{~mm}$ thickness were performed from the superior aspect of the horizontal portion of the mandible to the vertex as per the manufacturer's guidelines for image-guided acquisition. DCG was performed using the nonionic, water-soluble contrast medium (Iohexol, $755 \mathrm{mg} / \mathrm{mL}$ ). The contrast was diluted 50:50 with normal saline and injected slowly into the lacrimal drainage system with the help of lacrimal canula. Once CT-DCG images were acquired, the slices were reconstructed to sub-1 mm thickness, and 3D reconstruction was performed on the CT workstation after adjusting the Hounsfield values to detect the contrast density (Figure 1). Various scans were then uploaded to the navigation system and merged using the StealthStation Merger software to create a 3D model of the lacrimal drainage system for real-time tracking. The patient location was then registered and the navigation system could accurately demonstrate the location of an anatomical point radiologically in all the 3 CT-DCG planes.

\section{Results}

We illustrate 3 cases here to demonstrate the utility of DCG image guidance in complex lacrimal surgeries.

\section{Case I}

A female, aged 67 years, was referred for management of right-sided nasolacrimal duct obstruction (NLDO) following a total right-sided maxillectomy. She was a known case with maxillary sinus carcinoma and had undergone a right
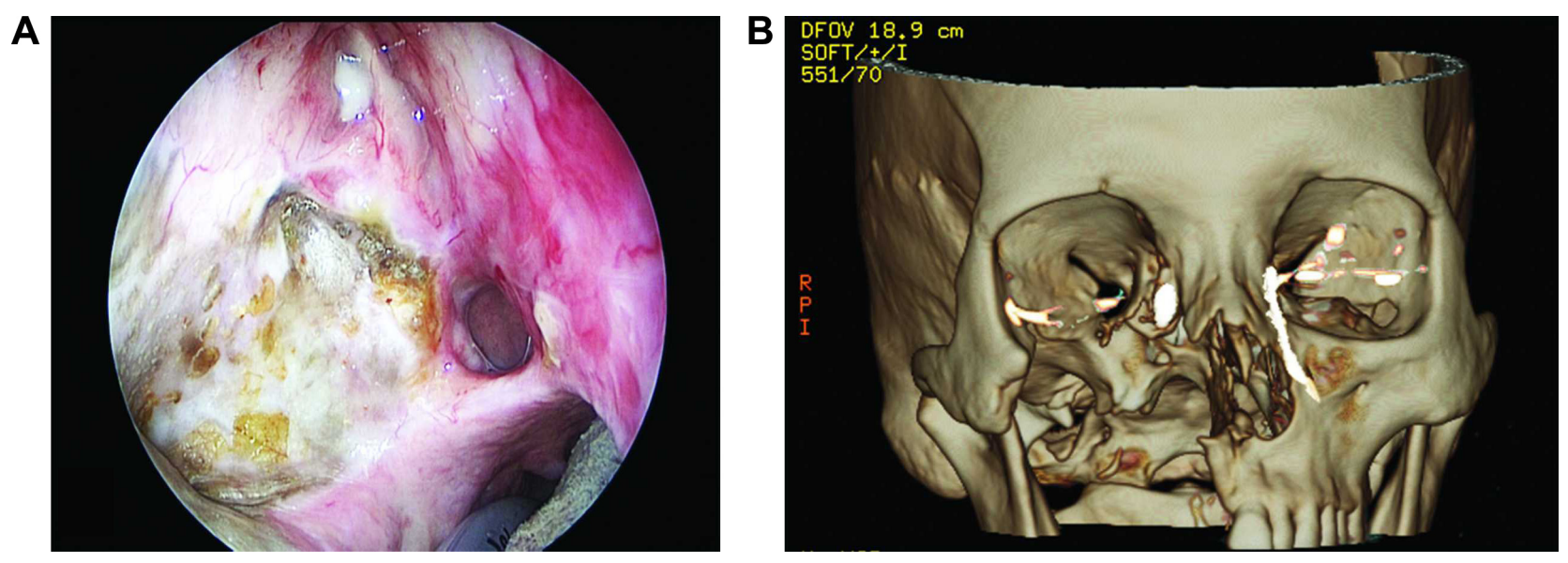

Figure I Case I: (A) Endoscopic photograph of the right nasal cavity showing the palatal defect, exposed bone of the lateral wall in the area of maxillary sinus and altered anatomy of the lateral wall. (B) The 3D CT-DCG showed absence of the right maxilla with right dilated lacrimal sac and an abrupt obstruction at the sac-duct junction. The DCG findings of the left lacrimal apparatus were normal.

Abbreviations: CT, computed tomography; DCG, dacryocystography. 
maxillectomy along with hemipalatal excision (Figure 1A) and was stable since then without any recurrence. She used a palatal prosthesis to prevent nasal regurgitation of oral contents during eating. The left lacrimal apparatus was normal. Endoscopic examination of the right nasal cavity showed gross alteration of the nasal anatomy (Figure 1A). There was no palpable bony lateral wall of the nose below the sac-duct junction. The area of the maxillary sinus was replaced by an exposed bone (Figure 1A) without a mucosal covering and a large hemipalatal defect (Figure 1A). The 3D CT-DCG showed absence of the right maxilla with right dilated lacrimal sac and an abrupt obstruction at the sac-duct junction (Figure 1B). The DCG findings of the left lacrimal apparatus were normal (Figure 1B). Navigation guidance using the CT-DCG was used to accurately delineate the boundaries of the dilated lacrimal sac (IGDL) (Figure 2) before proceeding with a continuous navigation-guided powered endoscopic DCR. Intraoperatively, the 3D-reconstructed CT-DCG virtual models were utilized (Figure 3 ) to constantly orient the surgeon to navigate in an altered anatomical milieu. There were no complications. The stereotactic image guidance in this case facilitated accurate localization of the obstructed lacrimal drainage apparatus, and this information helped the surgeon in precise planning and safe execution of the endoscopic surgery.

\section{Case 2}

A male, aged 42 years, presented with complaints of left-sided epiphora and discharge over 1-year duration. The complaints started after the patient sustained facial injuries with orbital fractures following a road traffic accident. Lacrimal evaluation showed a left-sided mucocele with regurgitation of mucoid material. The right lacrimal system was normal. Endoscopic examination of the left nasal cavity showed alteration of the nasal anatomy with axillary fracture of the middle turbinate and completely exposed maxillary sinus (Figures 4 and 5). The 3D CT-DCG showed an irregular dilated left lacrimal sac and an abrupt obstruction at the level of the proximal nasolacrimal duct (Figure 4B). The DCG findings of the right lacrimal apparatus were normal (Figure 4B). IGDL revealed useful clues on the altered location of the lacrimal sac in relation to the endoscopic lateral wall anatomy (Figure 5A). IGDL in combination with 3D CT-DCG provided precise geometric orientation during the continuous navigation-guided powered endoscopic DCR (Figure 5B).

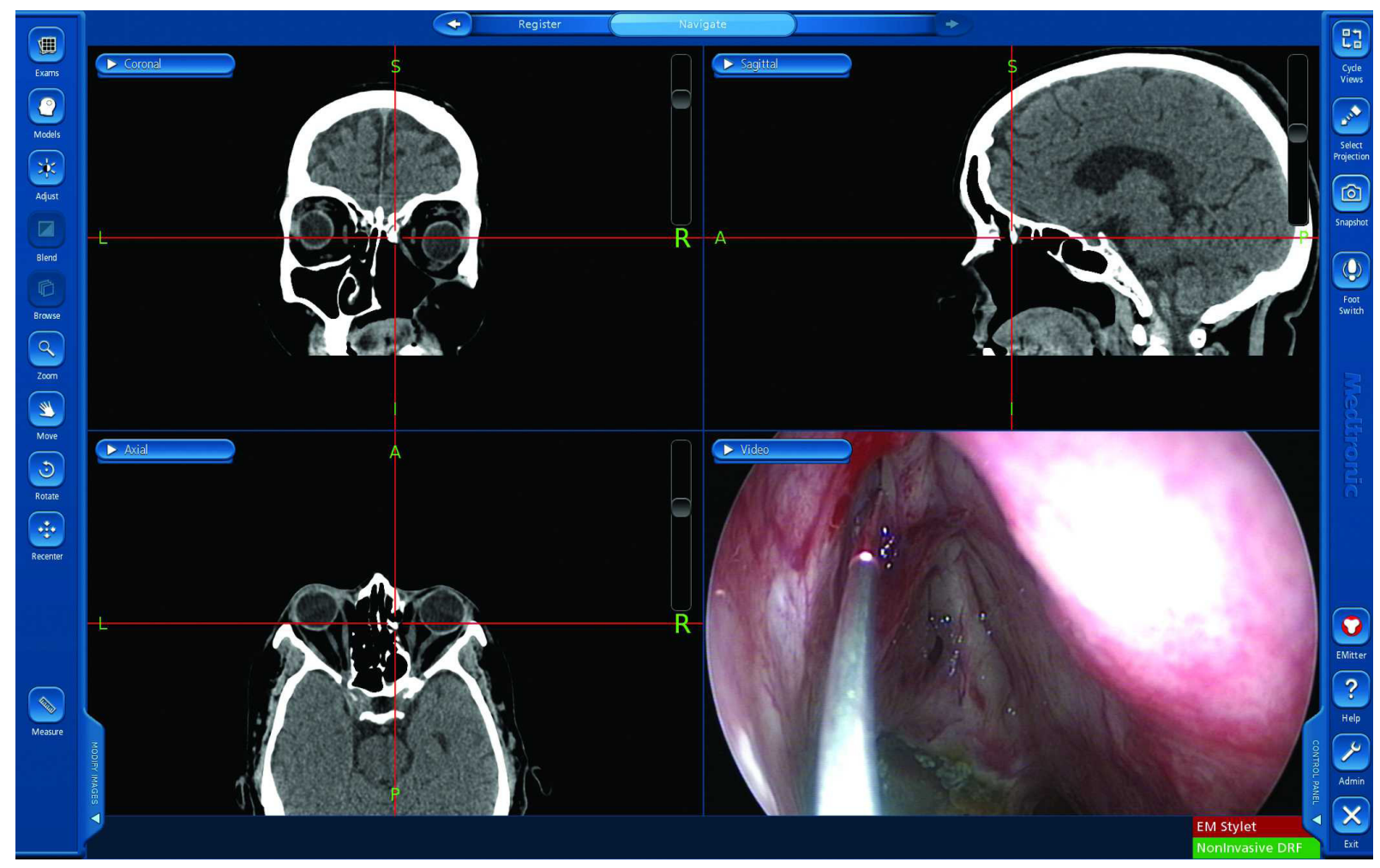

Figure 2 Case I: Image-guided dacryolocalization view showing the coronal (upper left), sagittal (upper right) and axial (lower left) CT images, as well as the endoscopic view (lower right).

Note: Note that the intersection of crosshairs shows the simultaneous endoscopic localization of the contrast-filled lacrimal sac.

Abbreviation: CT, computed tomography. 


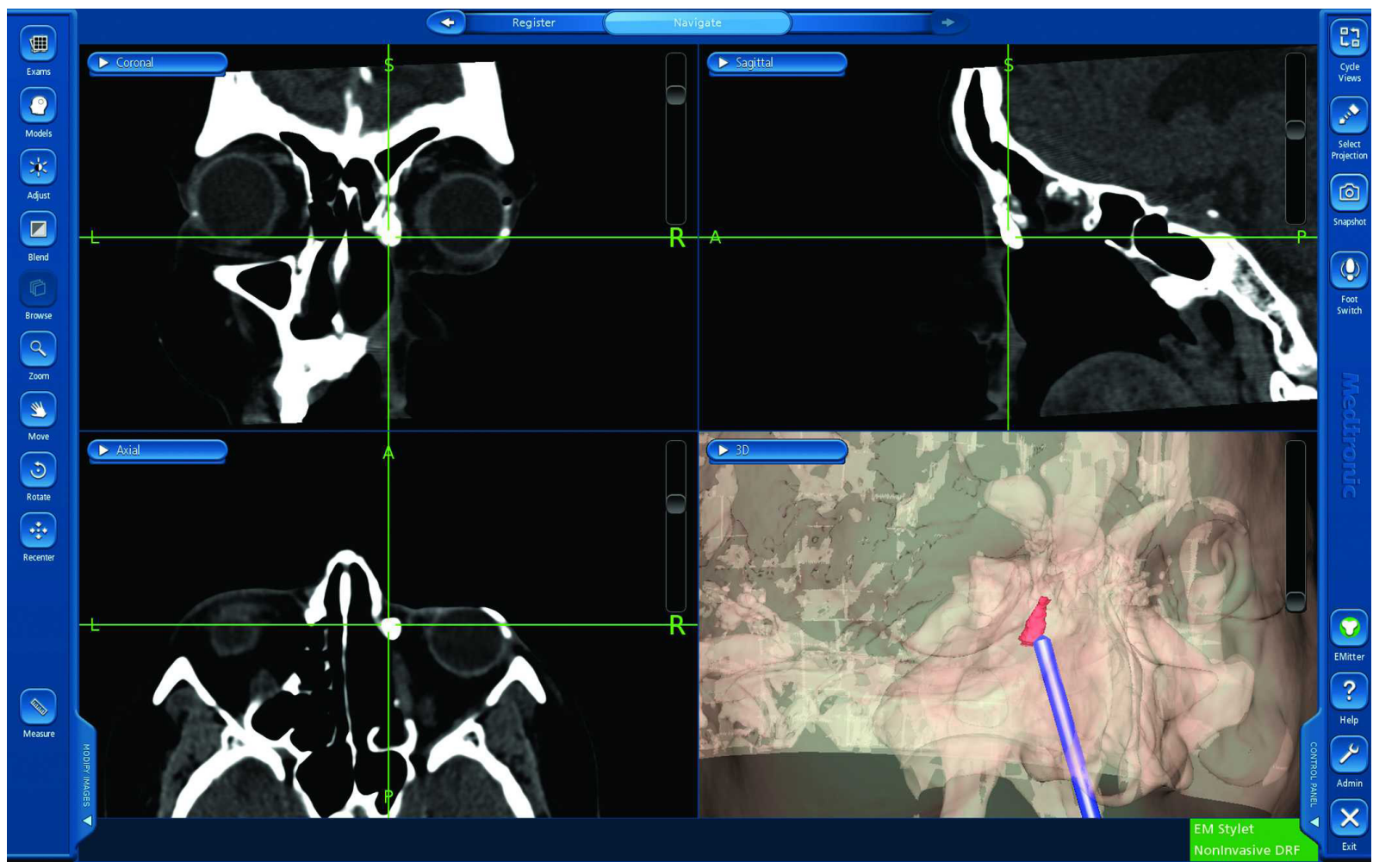

Figure 3 Case I: Intraoperative image-guided view depicting the image-guided dacryolocalization using the 3D-reconstructed virtual model of the DCG (lower right panel). Note: Note the sac being delineated as the red lesion being actively tracked by the blue endoscopic probe.

Abbreviation: DCG, dacryocystography.

\section{Case 3}

A male, aged 43 years, was referred for management of right-sided posttraumatic NLDO. The patient had rightsided epiphora and discharge over a duration of 2 years. The complaints started after the patient had a road traffic accident with resultant orbital fractures of the medial wall and floor. Lacrimal evaluation was suggestive of right complete
NLDO. The left lacrimal system was suggestive of a partial obstruction of the NLDO. The 3D CT DCG showed a regularly dilated right lacrimal sac and an abrupt obstruction at the level of the sac-duct junction (Figure 6A). The DCG findings of the left lacrimal apparatus showed multiple areas of stenosis at the sac-duct junction and within the nasolacrimal duct (Figure 6A). IGDL could obtain the precise location
A

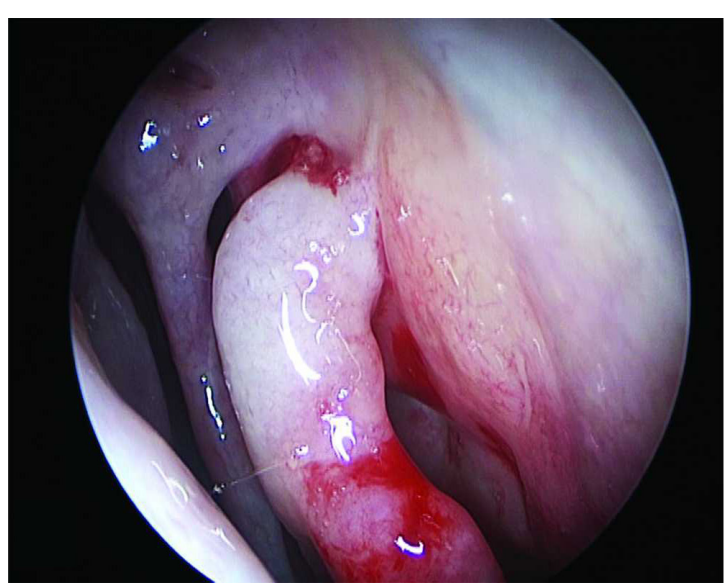

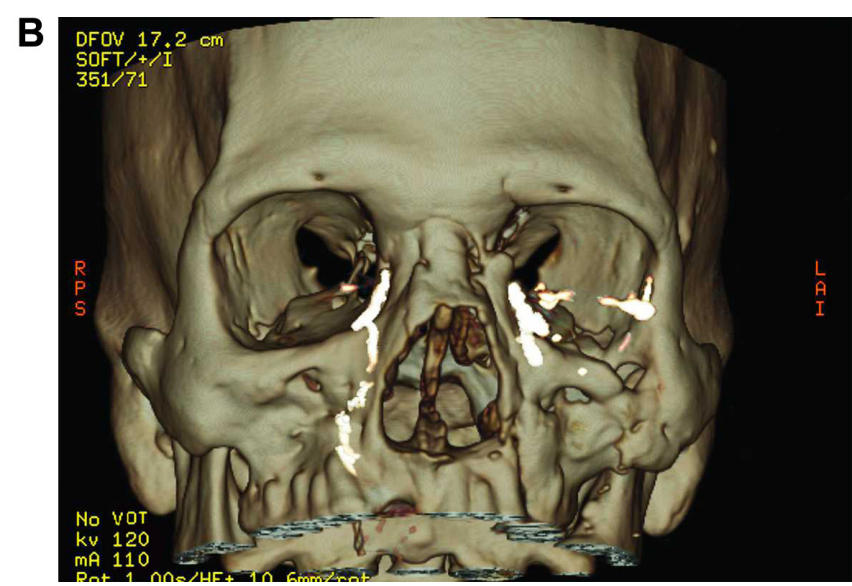

Figure 4 Case 2: (A) Endoscopic view of the left nasal cavity showing alteration of the nasal anatomy. (B) The 3D CT-DCG shows an irregular dilated left lacrimal sac and an abrupt obstruction at the level of the proximal nasolacrimal duct. The DCG findings of the right lacrimal apparatus were normal.

Abbreviations: CT, computed tomography; DCG, dacryocystography. 

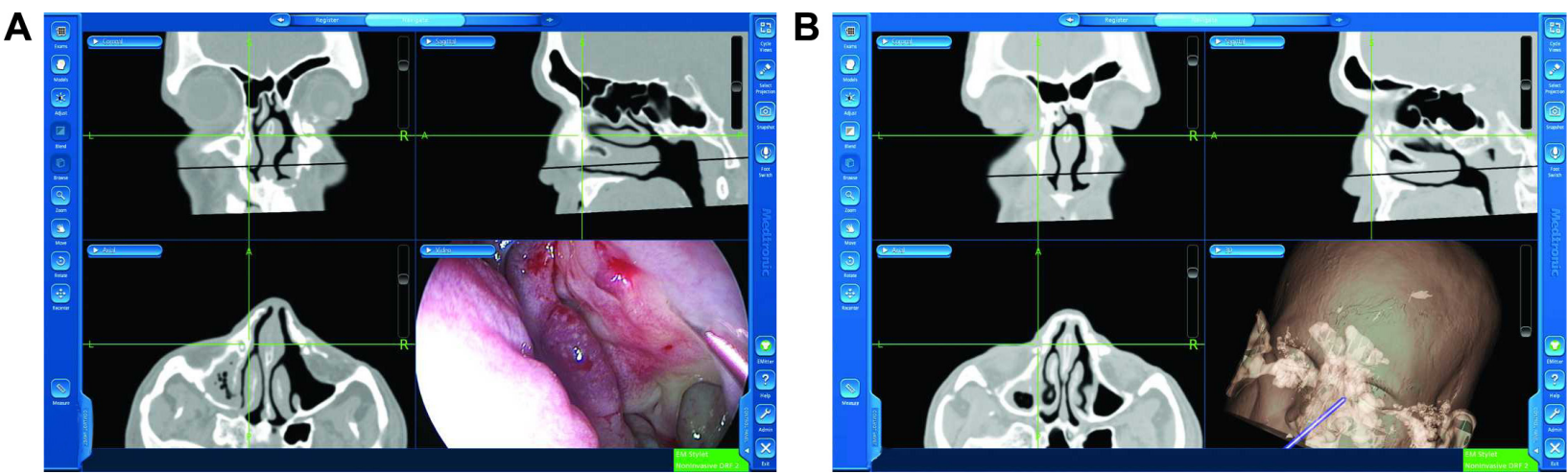

Figure 5 Case 2: (A) Image-guided dacryolocalization view showing the CT images and the endoscopic view (lower right). Note that the intersection of crosshairs shows the simultaneous endoscopic localization of the contrast-filled lacrimal sac. (B) Active endoscopic real-time tracking of the lacrimal apparatus using the 3D virtual model. Abbreviation: CT, computed tomography.

of the lacrimal sac in relation to the endoscopic lateral wall anatomy (Figure 6B). Following exposure of the presumed lacrimal tissues during the endoscopic DCR, intraoperative IGDL was able to successfully delineate the lacrimal apparatus from the surrounding areas of intense scarring (Figure 7). The surgery was completed safely without any complications.

\section{Discussion}

This study presents our preliminary report on image-guided lacrimal surgeries using 3D CT-DCG for stereotactic navigation. All the illustrated cases would have been difficult to operate by the conventional method with a mere CT scan as a guideline and, therefore, reflect the utility of DCG guidance in the management of complex secondary acquired lacrimal duct obstructions.
CT-DCG is one among the established modalities of imaging the lacrimal drainage apparatus and is known to help delineate the site of obstructions, differentiate between varying degrees of stenosis and aid in the detailed study of lacrimal mass lesions. Freitag et $\mathrm{al}^{4}$ showed that the 3D reconstruction of CT-DCG was superior to that using plain 2D CT-DCG because the entire column of the dye could be assessed in multiple planes. In addition, 3D-DCG was found to be useful in detecting subclinical partial obstructions, assessing the causes of a DCR failure and delineating the precise anatomy for better surgical planning in complex cases. The current series utilized these distinct advantages, in addition to the stereotactic or navigation-guided powered endoscopic DCR's benefits.

Navigation guidance for complex lacrimal surgeries using plain CT scan has been reported thrice earlier. Day et $\mathrm{al}^{1}$
A

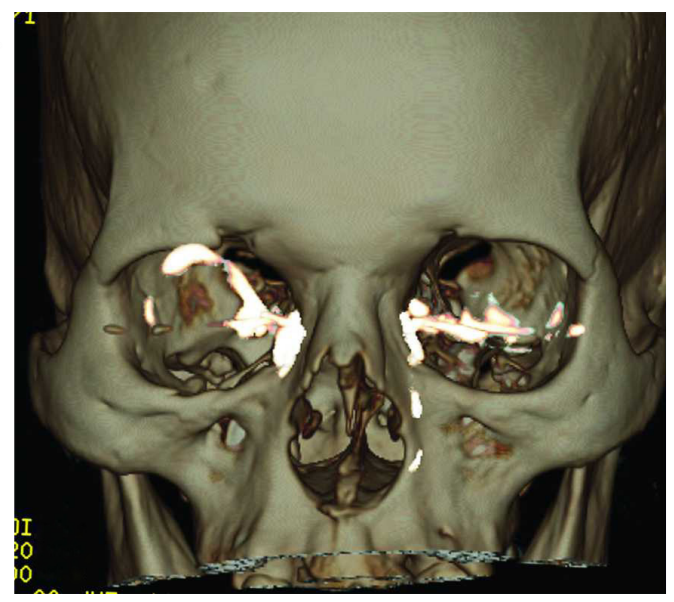

B

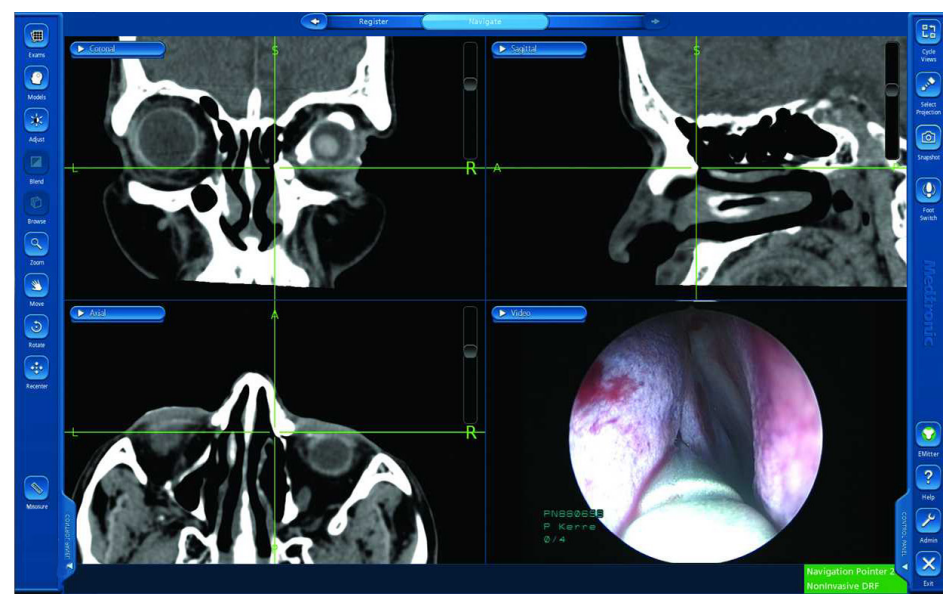

Figure 6 Case 3: (A) The 3D CT-DCG showed a regularly dilated right lacrimal sac and an abrupt obstruction at the level of the sac-duct junction. The DCG findings of the left lacrimal apparatus showed multiple areas of stenosis at the sac-duct junction and within the nasolacrimal duct. (B) Endoscopic localization of the contrast-filled sac. Note that the green crosshairs depict the accurate localization on all the CT images.

Abbreviations: CT, computed tomography; DCG, dacryocystography. 


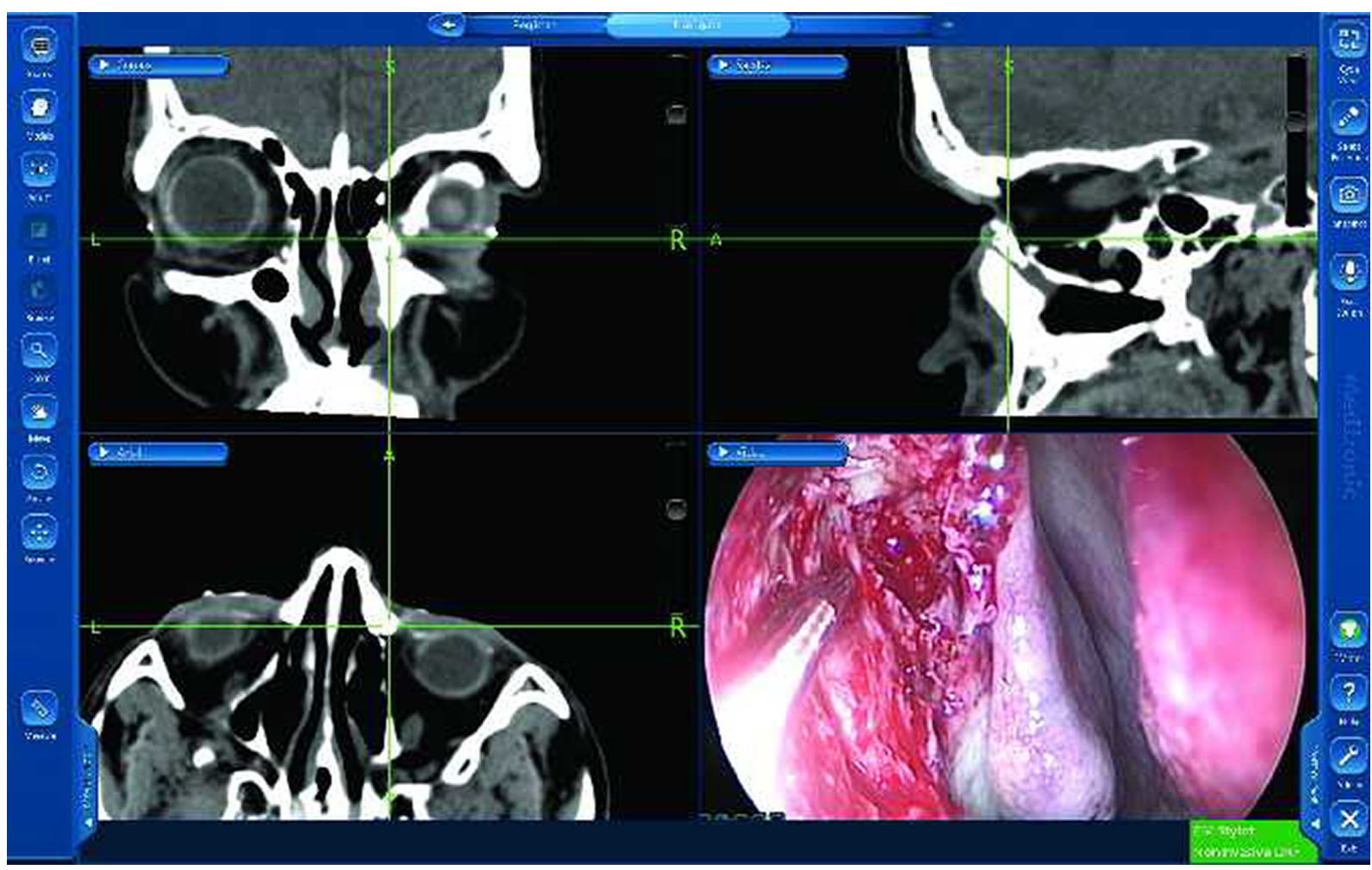

Figure 7 Case 3: Intraoperative active localization of the lacrimal apparatus following the osteotomy.

Note: Note the accurate localization in the sagittal plane depicting the geometric level of the probe and correlate that to the endoscopic tissue localization.

reported a 53-year-old man with bilateral NLDO secondary to cocaine abuse. The oronasal fistula, obliteration of meati and extensive endoscopic anatomical alteration necessitated the use of navigation guidance for the endoscopic DCR. Morley et $\mathrm{al}^{2}$ reported a 54-year-old man with left iatrogenic NLDO secondary to left rhinectomy and hemimaxillectomy for a sinonasal carcinoma. Navigation-guided placement of Lester Jones tube into the contralateral nasal cavity was successfully executed with the help of intraoperative navigation guidance. Ali et $\mathrm{al}^{3}$ coined the term "image-guided dacryolocalization" or IGDL to encompass the use of stereotactic navigation in lacrimal disorders. They reported 3 patients with gross nasoorbitoethmoid fractures with secondary acquired NLDOs. They showed that navigation guidance facilitates safe and precise surgeries in complex lacrimal disorders.

The limitations of these procedures could be the possible risk of iatrogenic trauma to the lacrimal apparatus or ocular irritation by the contrast medium while performing the CT-DCG; however, these were not noticed in the very limited experience from this series. There would also be an additional cost of acquiring 3D CT DCG scans, along with the added cost of navigation-guided surgical procedure. Hence, its utility of as now appears to be more cost-effective in complex cases as presented in this series.

\section{Conclusion}

Thus, 3D CT-DCG guided navigation can provide a useful radiological landmark while performing endoscopic DCRs in cases with secondarily acquired and complex NLDOs. Further large series, involving more varied etiopathologies, would help in formulating appropriate guidelines for intraoperative image guidance.

\section{Acknowledgments}

We acknowledge the help of Mr SBN Chary and Mr Gattu Naresh with photography and Ms Sabera Banu in the literature search and retrieval of the same. Support provided by The Operation Eyesight Universal Institute for Eye Cancer (SK), Hyderabad, India, is acknowledged. The funders had no role in the preparation, review or approval of the manuscript.

\section{Disclosure}

Mohammad Javed Ali receives royalties from Springer for his text book "Principles and Practice of Lacrimal Surgery". The other authors report no conflicts of interest in this work.

\section{References}

1. Day S, Hwang TN, Pletcher SD, et al. Interactive image-guided dacryocystorhinostomy. Ophthal Plast Reconstr Surg. 2008;24(4):338-340. 
2. Morley AM, Collyer J, Malhotra R. Use of an image-guided navigation system for insertion of Lester-Jones tube in a patient with disturbed orbito-nasal anatomy. Orbit. 2009;28(6):439-441.

3. Ali MJ, Naik MN. Image-guided dacryolocalization (IGDL) in traumatic secondary acquired lacrimal duct obstructions (SALDO). Ophthal Plast Reconstr Surg. 2015;31(5):406-409.

4. Freitag SK, Woog JJ, Kousoubris PD, Curtin HD. Helical computed tomographic dacryocystography with three dimensional reconstruction. A new view of the lacrimal drainage system. Ophthal Plast Reconstr Surg. 2002;18(2):121-132.
5. Ashenhurst M, Jaffer N, Hurwitz JJ, Corin SM. Combined computed tomography and dacryocystography for complex lacrimal problems. Can J Ophthalmol. 1991;26(1):27-31.

6. Caldemeyer KS, Stockberger SM, Broderick LS. Topical contrast enhanced CT and MR dacryocystography: imaging the lacrimal drainage apparatus of healthy volunteers. AJR Am J Roentgenol. 1998; 171(6):1501-1504.

7. Mannor GE, Millamn AL. The prognostic value of preoperative dacryocystography in endoscopic transnasal dacryocystorhinostomy. Am J Ophthalmol. 1992;113(2):134-137.
Clinical Ophthalmology

\section{Publish your work in this journal}

Clinical Ophthalmology is an international, peer-reviewed journal covering all subspecialties within ophthalmology. Key topics include: Optometry; Visual science; Pharmacology and drug therapy in eye diseases; Basic Sciences; Primary and Secondary eye care; Patient Safety and Quality of Care Improvements. This journal is indexed on

Submit your manuscript here: http://www.dovepress.com/clinical-ophthalmology-journal

\section{Dovepress}

PubMed Central and CAS, and is the official journal of The Society of Clinical Ophthalmology (SCO). The manuscript management system is completely online and includes a very quick and fair peer-review system, which is all easy to use. Visit http://www.dovepress.com/ testimonials.php to read real quotes from published authors. 